\title{
3D Measurements of Wear on Machining Tools Using a Confocal Microscope
}

S. Huq*, B. Abidi*, C. Kammerud, D. Page*, and M. Abidi*

J. Frafjord** and S. Dekanich**

*The University of Tennessee, 1508 Middle Drive, Knoxville, TN 37996

**Y-12 National Security Complex, Bear Creek Rd, Oak Ridge, TN 37831

The three dimensional (3D) reconstruction of a machining tool is carried out using Confocal microscope scans. It is then demonstrated that the volume of the worn out scraps from the tool after its use in machining, can be recovered using the 3D reconstruction of the used tool. Partial 3D models are first obtained at given orientations of the tool and then combined to generate the overall 3D model of the region of interest. The 3D model is then aligned with and subtracted from the standard model (CAD for example) of the tool before usage. The subtraction provides the missing scraps of the tool that were lost to wear or incurred by other types of damage.

Confocal Microscope Scanning is an active method for 3D modeling. The microscope uses a laser line to illuminate and then image the object at different micro/nano level depths of view. Each scan focuses only on a narrow slice of the object. Surfaces above and below the slice appear as a blurred background. Fig. 1 shows the tool being characterized and few frames of a confocal scan series. The slice location where a point is at its maximum brightness indicates the depth value of that point. A series of scans provides a series of planes and their depth information. When combined, the series gives the depth map of the points on the scanned surface. Gaussian mask or non-linear curve fitting are used to extract the exact depth value.

To observe wear and tear on its surface, the tool was scanned from different directions so that all the areas in the region of interest can be covered. Partial 3D models are obtained from each stack of scans and then combined together to obtain a full 3D model of the region of interest (Fig. 2 and 3). The scans were all taken at $10 \mathrm{X}$ magnification with a fixed scan grid resolution of $512 \times 512$. With higher magnification and higher resolution, smaller missing parts can be extracted and higher accuracy of volume computation can be achieved. The volume of the 3D model shown in Fig. 4(d) was estimated at $0.022 \times 10^{9} \mu \mathrm{m}^{3}$ using a Monte Carlo Method as described in [1].

References

[1] Yong Tsui Lee and Aristides G. Requicha, Communications of the ACM. 25 (9), (1982) 635641.

This work was supported by the DOE University Program in Robotics grant DOE-DE-FG522004NA25589.

The tool was provided by Y-12 National Security Complex. 


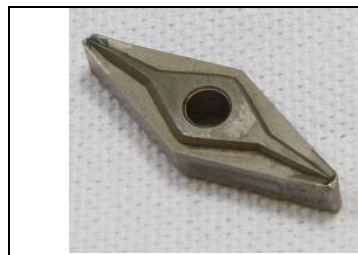

(a)

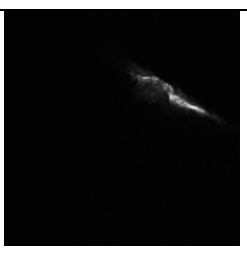

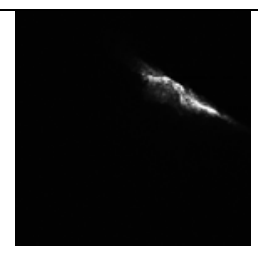

2

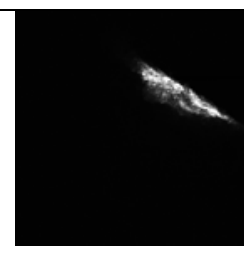

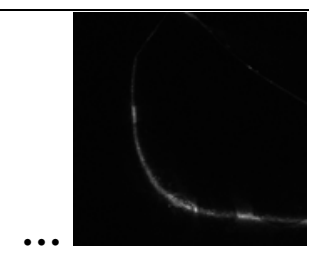

464

(b)

Fig. 1. (a) Tool to be characterized; (b) scans from a 464 slice stack each of resolution $512 \times 512$.

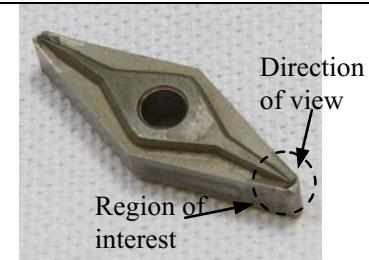

(a)

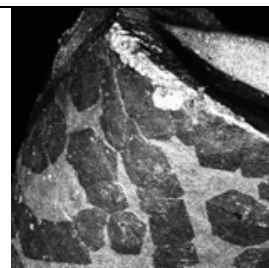

(b)

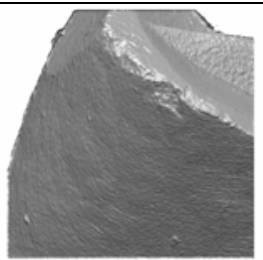

(c)

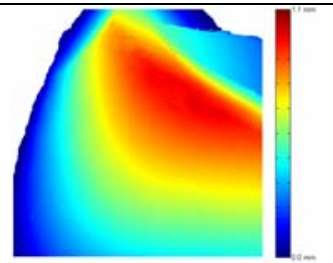

(d)

Fig. 2. Partial 3D model; (a) Tool; (b) textured partial model using the green channel; (c) Shaded partial 3D model; (d) Color-coded partial 3D model.

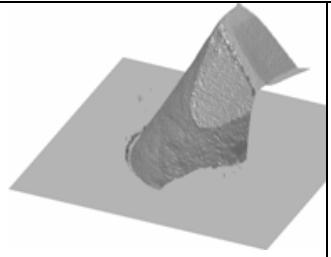

(a)

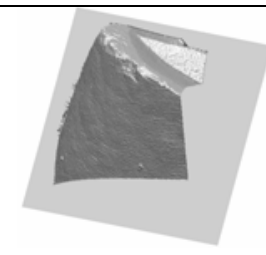

(b)

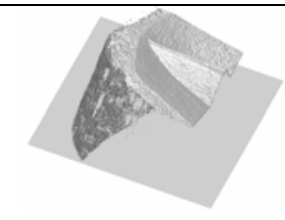

(c)

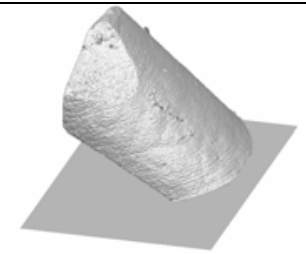

(d)

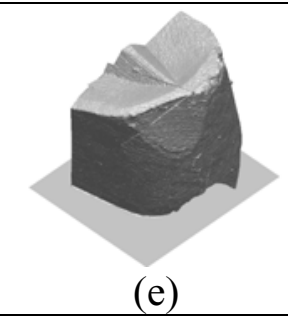

(e)

Fig. 3. (a), (b), (c), and (d) partial 3D models from four different sides of the region of interest of the tool as in Fig. 2 (a); (e) Registered 3D model obtained by combining (a), (b), (c), and (d).

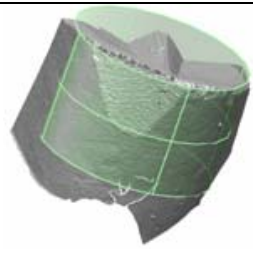

(a)

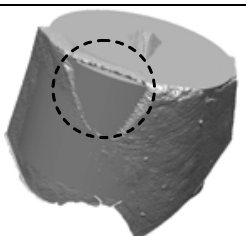

(b)

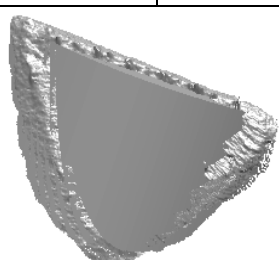

(c)

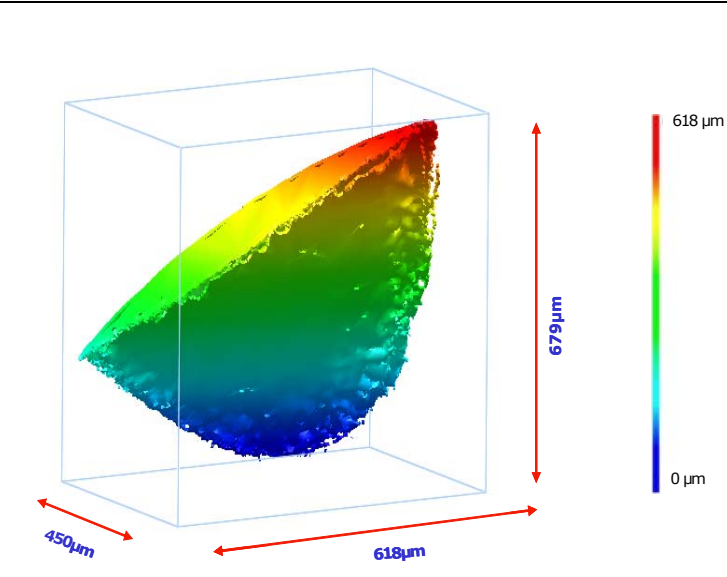

(d)

Fig. 4. (a) Perfect cylinder (or CAD when available) superimposed on reconstructed model of the used tool in Fig 3(e); (b) Cylinder registered; (c) Zoomed-in volume difference or missing scrap; (d) Color coded missing scrap in a bounding box of size $450 \times 618 \times 679 \mu \mathrm{m}^{3}=0.188 \times 10^{9} \mu \mathrm{m}^{3}$. The actual scrap volume is $0.022 \times 10^{9} \mu \mathrm{m}^{3}$ 